\title{
Sedimentation of Halloysite Nanotubes from Different Deposits in Aqueous Media at
}

\section{Variable Ionic Strengths}

Giuseppe Cavallaro $^{\mathrm{a}, \mathrm{b}}$, Giuseppe Lazzara ${ }^{\mathrm{a}, \mathrm{b}}$, Vincenzo Taormina ${ }^{\mathrm{a}}$, Donato Cascio $^{\mathrm{a}}$

${ }^{a}$ Dipartimento di Fisica e Chimica, Università degli Studi di Palermo, Viale delle Scienze, pad. 17, 90128 Palermo, Italy.

${ }^{b}$ Consorzio Interuniversitario Nazionale per la Scienza e Tecnologia dei Materiali, INSTM, Via G. Giusti, 9, I-50121 Firenze, Italy

\begin{abstract}
Halloysite clay is a natural nanomaterial that is attracting a growing interest in colloidal science. The halloysite aqueous dispersion stability is a key aspect for the configuration of a purification protocol as well as to establish the durability of a formulation. A physicochemical study demonstrated the role of ionic strength and nanotube characteristic sizes on the sedimentation behavior. We highlighted the importance of the electrostatic repulsions exercised between the particles in the settling process. A protocol for image analysis has been proposed to provide robust information from time resolved optical images on the suspensions. In conclusion, we managed to correlate microscopic aspect to the peculiar sedimentation process of halloysite nanotubes.
\end{abstract}

KEY WORDS. Halloysite, sedimentation, image analysis, $\zeta$ potential, diffusion. 


\section{Introduction}

The use of clay minerals is rising up in materials during recent decades due to their environmental-friendly nature, and availability in different shape and characteristic sizes[14]. The sedimentation of solid clay micro and nanoparticles in aqueous media has been attractive for scientists due to many practical applications such as stability of dispersions and separation or purification strategies[5-11]. Typically, clay nanoparticles possess a nanolayered morphology that, in mesoscopic scale, can evolve to anisotropic nanoparticles such hollow tubular shape [12]. Natural halloysite nanotubes (HNTs) are promising nanocontainers with controlled release properties that make them attractive for smart formulations with encapsulated active molecules[13-16]. HNT generates by rolling-up a kaolin double layer forming a tubular structure of ca. $1 \mu \mathrm{m}$ in length and outer radius ranging between 15 to $100 \mathrm{~nm}$ [17].

HNTs are available in caves worldwide but each source is characterized by mineral purity and nanotube aspect ratios that strongly influence the physico-chemical properties of this nanomaterial such as loading ability and strengthener action when used as inorganic filler in polymeric matrices [18-22]. Therefore, separation and purification methods based on sedimentation in aqueous media can be a key pretreatment to prepare a reproducible nanomaterial with controlled sizes and properties.

Halloysite nanotubes have been proposed for a large variety of industrial applications: in healing anticorrosion [23-25], drug delivery [14,16,26-31], conservation of Cultural Heritage [32-36] and support for catalysis [37-43]. For all uses in liquid formulations, the nanoparticle dispersion stability is a crucial aspect and it can be improved, or controlled, by polymeric or small molecules as additives [44-46]. As concerns the colloidal stability of HNTs in aqueous dispersion, a peculiar sedimentation behavior has been observed in 1952 showing the formation of a large sedimentation volume [47]. Recently, some efforts have been addressed 
in highlighting the interesting behaviors in concentrated dispersion that allows to liquid crystalline and phases with long-range order parameters [48]. Several surface modifications have been investigated by adding ionic polymers $[45,49]$ and surfactants $[50,51]$ to control the halloysite stability in aqueous media and to induce the nanotubes alignment [46].

This study aims at elucidating the effects on halloysite sedimentation of ionic strength by changing the sodium chloride concentration. To achieve this goal, a series of sedimentation tests with time lapsed optical recording were performed. A method for the identification and analysis of the motion of the sedimentation front is presented. The automatic sedimentation front detection presents numerous difficulties, essentially due to:

- low contrast between the separation zones of the sedimentation front;

- the curvature of the front near the walls of the container, generated by the forces of liquid wall cohesion, makes it difficult to identify the line that best represents the sedimentation front position.

In the present work, the problem of sedimentation front detecting has been addressed by developing an advanced method of image processing. In particular, a Sobel derivative filter for signal detection was implemented, morphological filters for noise suppression were used, and the system was based on the use of the Hough line transform [52].

$\zeta$-potential and dynamic light scattering yields insight on the inter-particle forces and diffusion dynamics of halloysite in dilute regimes. These results are essential for a correct evaluation of the interaction between the clay nanoparticles and the interpretation of the sedimentation experiments in terms of sedimentation volume and settling rate dependence on ionic strength. 


\section{Materials and methodology}

Halloysite nanotubes from two geological deposits have been employed. In particular, HNT from Dragon Mine Utah (HNT_DG) that was a Sigma product and HNT from Matauri Bay in New Zealand (HNT_NZ) kindly provided by Imerys Ceramics. Table 1 reports the main characteristics of Halloysite samples and Figure 1 reports SEM images for the investigated clay nanotubes. $\mathrm{NaCl}(>99 \%)$ was a Sigma product. Water from reverse osmosis (Elga model Option 3) with a specific resistivity greater than $1 \mathrm{M} \Omega \mathrm{cm}$ was used.

Table 1. Characteristics of halloysite samples in this study.

\begin{tabular}{|c|c|c|}
\hline Sample code & HNT_DG & HNT_NZ \\
\hline Geological deposit & Dragon Mine & Matauri Bay \\
\hline Locality & Utah, North America & Northland, New Zealand \\
\hline Surface area $\mathrm{a}^{\mathrm{a}} / \mathrm{m}^{2} \mathrm{~g}^{-1}$ & 46.4 & 28.6 \\
\hline External radius ${ }^{b} / \mathrm{nm}$ & 10 to 75 & 25 to 100 \\
\hline Tube Length ${ }^{\mathrm{b}} / \mu \mathrm{m}$ & 50 to 1500 & 100 to 3000 \\
\hline
\end{tabular}

${ }^{\mathrm{a}}$ From ref [53]. ${ }^{\mathrm{b}}$ From ref [17].
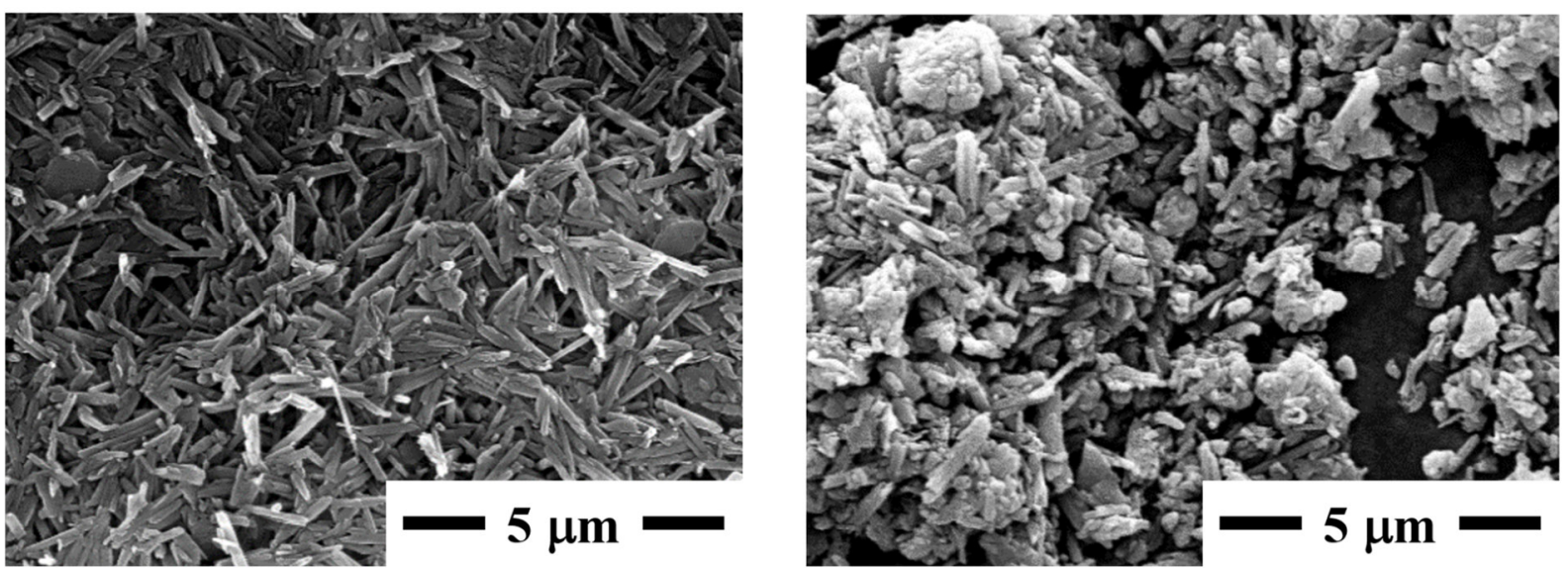

Figure 1. SEM images for HNT_NZ (left hand side) and HNT_DG (right hand side) samples 
2.1 Preparation of HNTs dispersions. Halloysite nanotubes were added to water and $\mathrm{NaCl}$ aqueous solution. The obtained dispersions were sonicated for $15 \mathrm{~min}$ and magnetically stirred for 3 days to ensure the full hydration of the clay particles.

\subsection{Methods.}

$\zeta$-potential and dynamic light scattering (DLS) measurements were carried out by means of a Zetasizer NANO-ZS (Malvern Instruments). The field-time autocorrelation functions were described by a single decay, which provides the decay rate $(\Gamma)$ of the diffusive mode. For the translational motion, the collective diffusion coefficient at a given concentration is $D_{t}=\Gamma / q^{2}$ where $\mathrm{q}$ is the scattering vector given by $4 \pi \mathrm{n} \lambda^{-1} \sin (\theta / 2)$ being $\mathrm{n}$ the water refractive index, $\lambda$ the wavelength $(632.8 \mathrm{~nm})$ and $\theta$ the scattering angle $\left(173^{\circ}\right)$.

The functionalized nanotubes were imaged by using a microscope ESEM FEI QUANTA 200F. Before each experiment, the sample was coated with gold in argon by means of an Edwards Sputter Coater S150A to avoid charging under electron beam.

The sedimentation experiments were carried out in glass tubes of ca. $2 \mathrm{~cm}$ in diameter and length of ca. $6 \mathrm{~cm}$. The dispersions were imaged every $10 \mathrm{~min}$ until equilibration in vertical position.

\subsection{Image analysis and sedimentation fronts detection}

The sedimentation front identification in the sequence of images acquired at constant time intervals was obtained with algorithms of image processing. For the development of the method, the functions in the OpenCV open source library have been used (site: https://opencv.org/). The proposed method consists of three phases:

1. Image preprocessing: are applied transformations with the objective, the improvement of the signal to noise ratio, and the standardization of the images; 
2. Regions of Interest (ROIs) extraction: the regions (inside the vials) in which the liquid is present are identified in order to limit the analysis of the successive phase and to allow the determination of the height of the sedimentation front;

3. Best line identification: the ROIs are analyzed and for each one the straight line that best describes the sedimentation front is identified.

\subsubsection{Image preprocessing}

In this phase, image transformations are carried out in order to make the subsequent identification steps more effective. An intensive analysis of the preprocessing function was conducted, aimed at maximizing the performance. In particular, this analysis led to the selection of the following preprocessing operations[54]:

1) grayscale image conversion;

2) application of a Gauss filter with $5 \times 5$ kernel for noise reduction;

3) image stretching;

Figure 2 shows an example of image before and after the preprocessing phase.
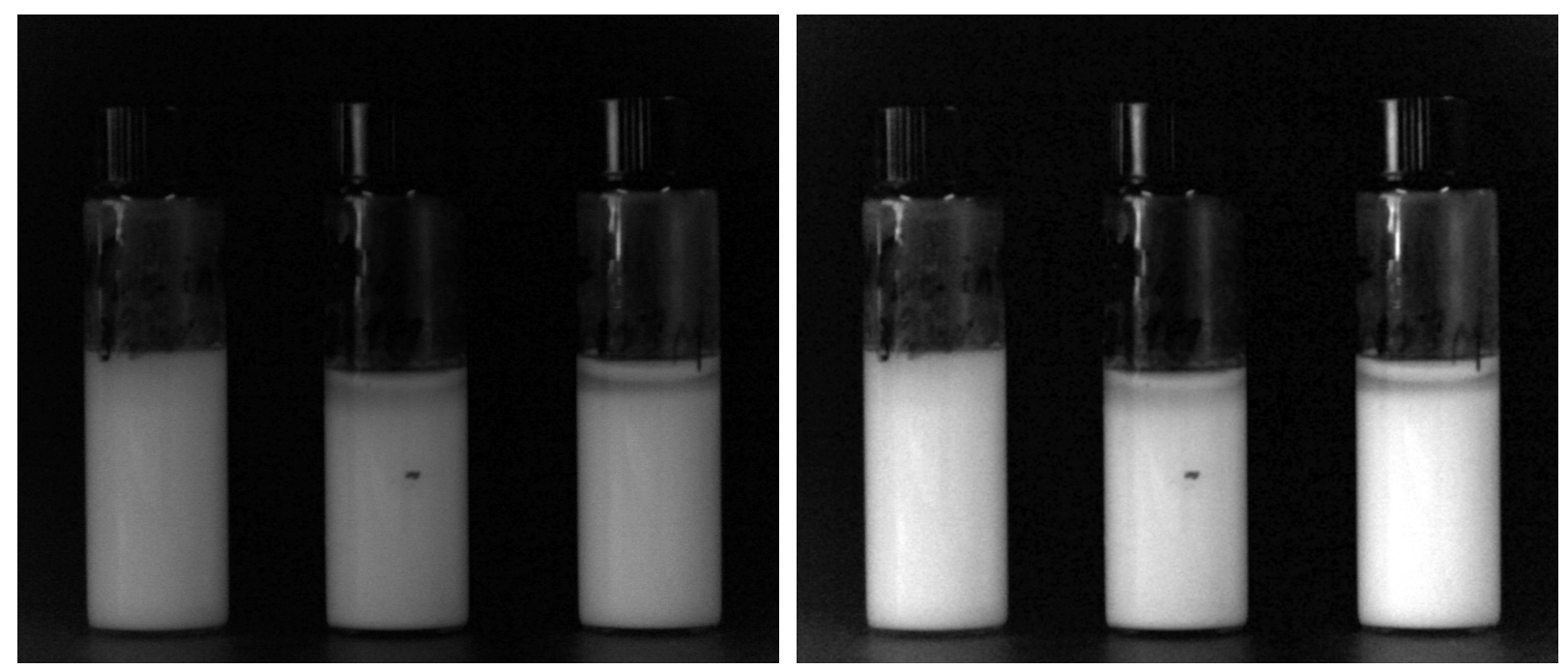

Figure 2. Example of an image to be analyzed (left hand side), relative preprocessed image (right hand side). 


\subsubsection{ROIs extraction}

This phase of the method involves the identification of the regions of interest, ie the determination of the three areas of the image, contained within the vials, where the liquid is present and the sedimentation front of which the motion is to be analyzed. The steps implemented for the extraction of ROIs are the following:

1) Otsu thresholding and image binarization: the optimal intensity threshold value is identified; all pixels with an intensity value below the threshold will be zeroed. Subsequently a binarization is carried out, ie the pixels of intensity different from zero are forced to the maximum value (255).

2) filling and small regions removal: the regions found, as they could contain gaps, are filled. In addition, the small area regions are suppressed.

3) find contours and boundary box: the outline of the region is identified and finally the rectangle that surrounds it is found.

Figure 3 shows the sequence of processing results obtained on an example image.

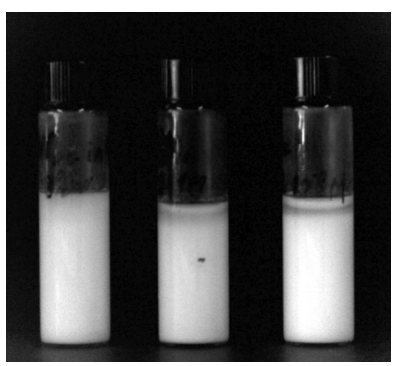

(a)

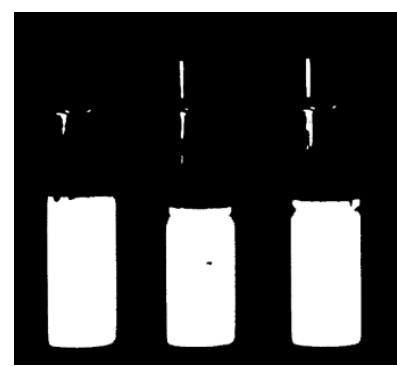

(b)

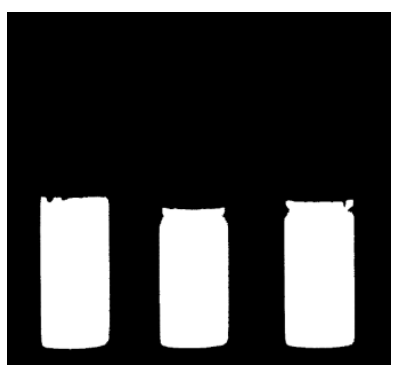

(c)

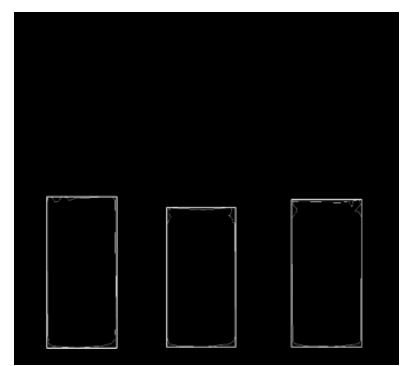

(d)

Figure 3. Example of ROIs detection: a) preprocessed image, b) binary image after the thresholding, c) image after filling and small regions removal, d) image with highlighted rectangles, obtained after the find contour and the boundary box, which delimit the regions of interest

\subsubsection{Best line identification}

The problem of determining the sedimentation front has been addressed by developing a filter designed to use the following specific information: 
- the sedimentation front delimits two regions of space, the lower one presenting greater intensity;

- the sedimentation front can be represented, especially in the most central area of the vial (away from the walls), by a horizontal line.

These considerations have led us to implement a Sobel derivative filter (kernel 5x40), with only a vertical component (the intensity transition occurs from top to bottom). To improve detection efficiency, the filter was applied only on a width equal to half the width of the generic ROI and in particular in the central part of the ROI; in this way a front was not sought in proximity to the walls of the vial where the effects of curvature decrease the effectiveness of the filter. The filtering result was normalized (saturating at 1\%). A subsequent morphological erosion filter is applied in order to eliminate small groups of pixels that can not be associated with a sedimentation front. The steps described are to prepare the image for the execution of the horizontal segment search algorithm that best describes the position of the sedimentation front. For this purpose, it was decided to use a probabilistic Hough line transform [52].

The Hough transform is a feature extraction technique used in image analysis [55-57]. The purpose of the technique is to find imperfect instances of objects within a certain class of shapes by a voting procedure. This voting procedure is carried out in a parameter space, from which object candidates are obtained as local maxima in a so-called accumulator space that is explicitly constructed by the algorithm for computing the Hough transform. The classical Hough transform was concerned with the identification of lines in the image. The search for lines in this work has been limited to small angles, as we look for a horizontal representation of the front.

Figure 4 shows an example of ROI analysis and consequent identification of the sedimentation front. 


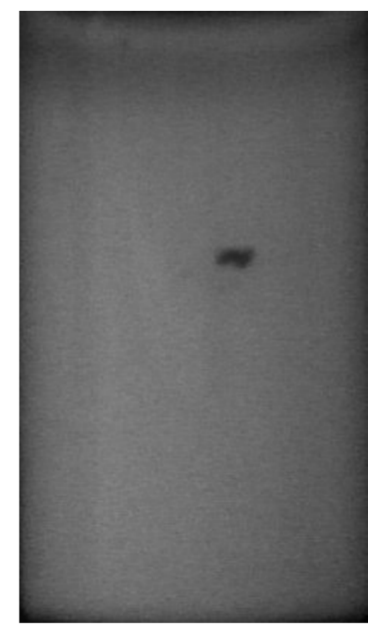

(a)

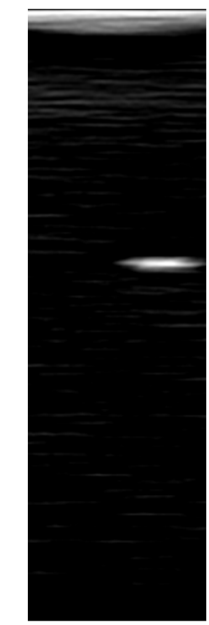

(b)

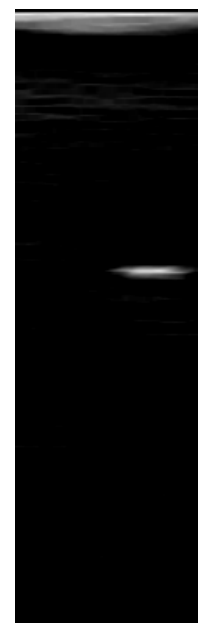

(c)

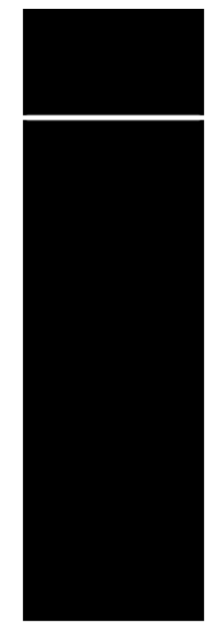

(d)

Figure 4. Example of sedimentation front detection: a) preprocessed image, b) image after Sobel filter and normalization, c) image after erosion filter, d) best straight line obtained from the Hough line transform

\section{Results}

The colloidal dispersion of HNT in water was characterized in a dilute regime $\left(\mathrm{C}_{\mathrm{HNT}}=0.01\right.$ $\mathrm{wt} \%$ ) as a function of the $\mathrm{NaCl}$ concentration (from $10^{-4}$ to $1 \mathrm{M}$ ). The apparent hydrodynamic radius was plotted as a function of the square root of ionic strength in Figure 5. Regardless of the specific Halloysite nature, in both cases, a slowdown of the translational diffusion dynamic is observed in the presence of salt and this effect is more pronounced for the HNT_NZ sample. Going further, it should be noted that the lowest investigated $\mathrm{NaCl}$ concentration did not alter significantly the Rh values. 


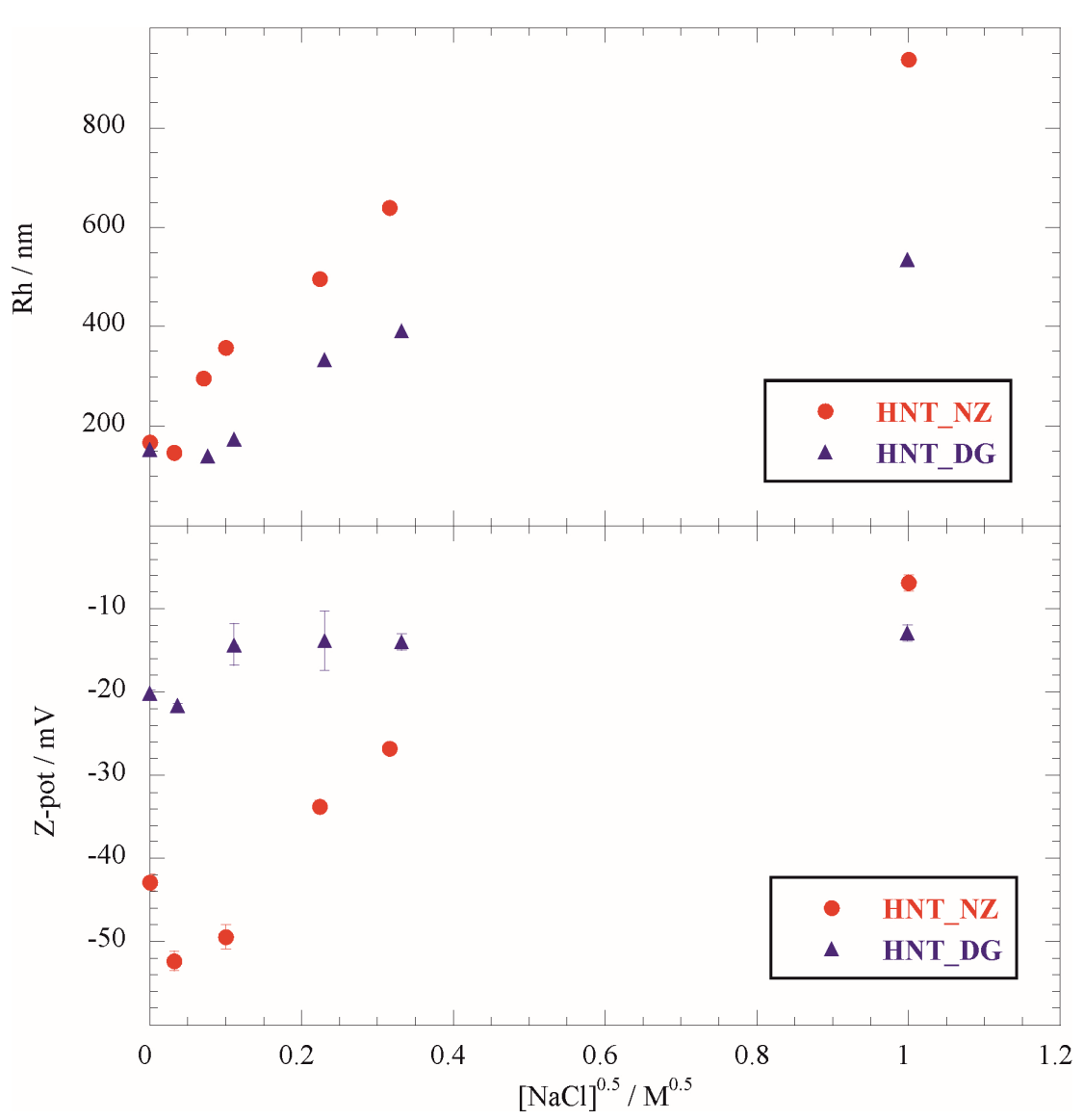

Figure 5. Hydrodynamic radius and $\zeta$-potential for HNTs samples as a function of the square root of ionic strength.

As concerns the surface charge of the nanotubes, it was investigated by monitoring the $\zeta$ potential in $\mathrm{NaCl}$ aqueous solutions (Figure 5). Large salt concentration generates a variation of the $\zeta$-potential values towards smaller effective charges. Similarly, to dynamic light scattering evidences, low $\mathrm{NaCl}$ concentration generated an opposite effect than in high ionic strength conditions being that the $\zeta$-potential values are slightly more negative than those of the corresponding systems in water are. The $\zeta$-potential data evidenced that HNT_NZ is more sensitive to salt addition than the HNT_DG. The larger negative $\zeta$-potential values of HNT_NZ compared to HNT_DG is in agreement with the literature reports [53]. 


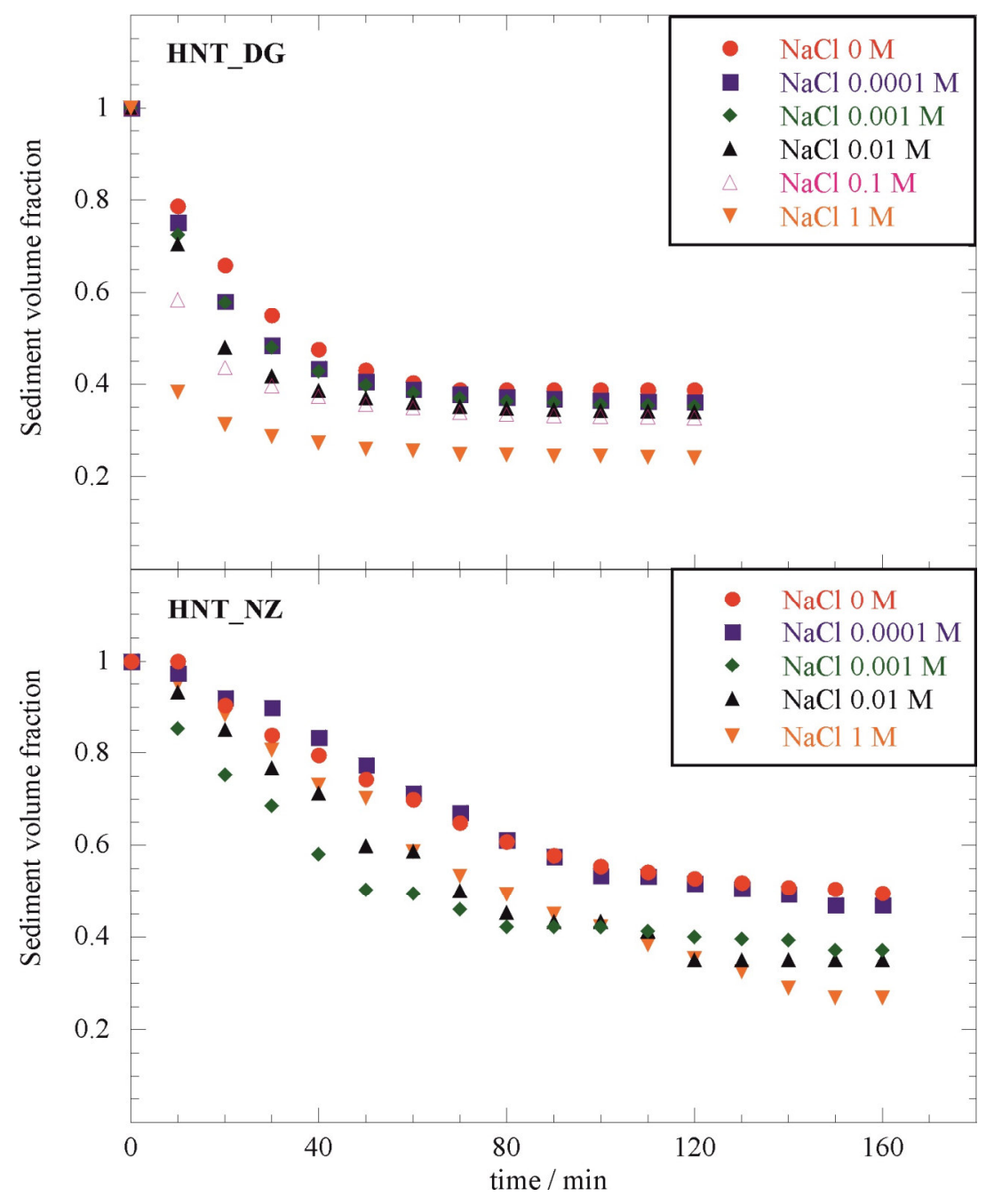

Figure 6. Time dependence of sediment volume fraction obtained from time-lapse experiment and image analysis for front detection

The sedimentation experiments were carried out in concentrated HNT conditions $(4.0 \mathrm{wt} \%)$. In general, a sedimentation of the clay particles is imaged with a certain settling rate that after ca. 1 hour slows down approaching a plateau (Figure 6). In particular, after equilibration two phases were observed, an upper transparent aqueous phase and a lower milky phase with a high clay content. These measurements after automated image analysis provided two main insights including the settling velocity of the clay front and the residual sedimentation volume fraction (Figure 7). The sedimentation volume decreased with the increment of ionic strength 
for both HNTs samples. Differently, the settling velocity for HNT_NZ is not significantly influenced by $\mathrm{NaCl}$ whilst sedimentation is speedup by the salt addition for HNT_DG.

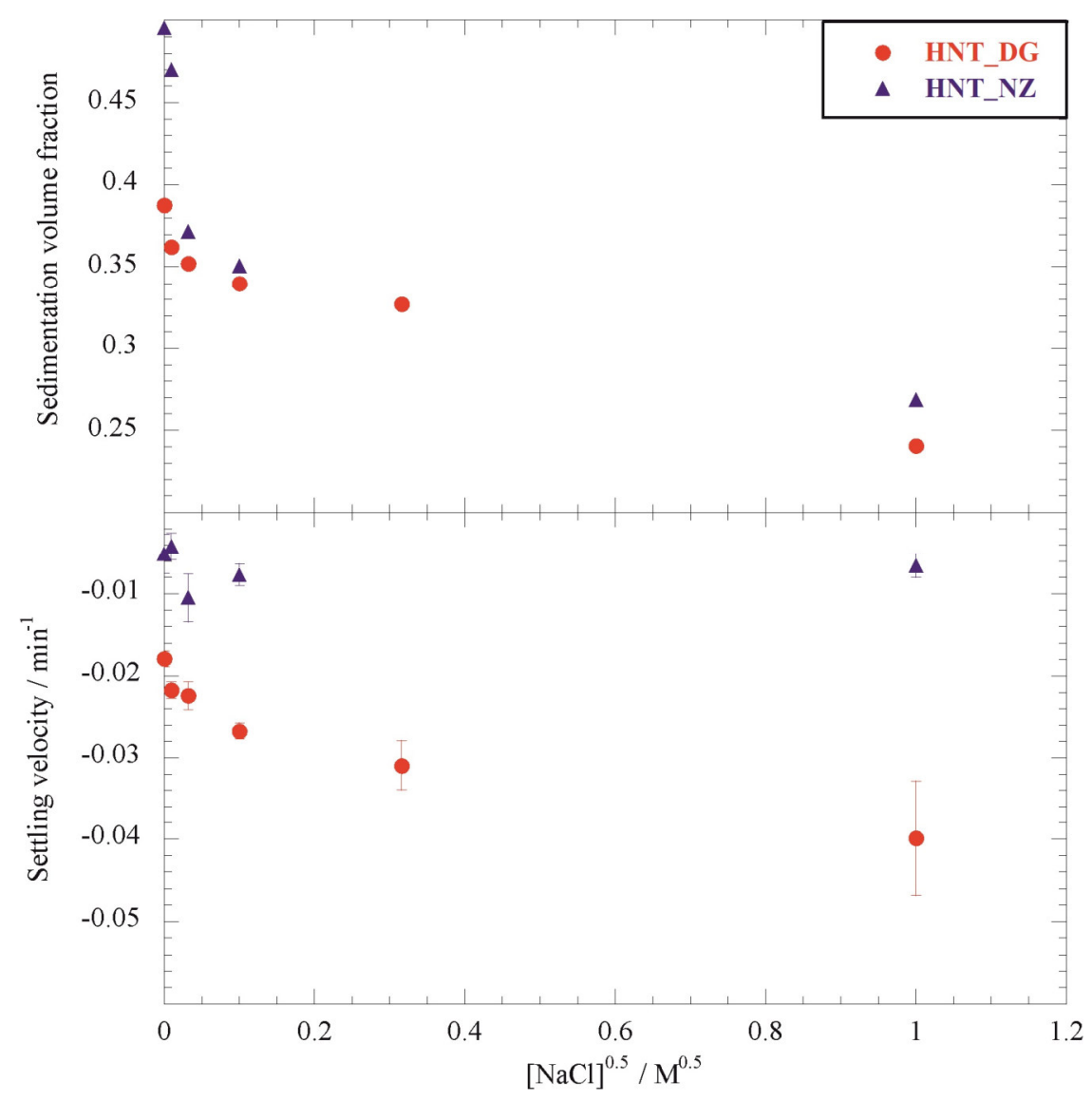

Figure 7. Settling velocity and sedimentation volume fraction for HNTs samples as a function of the square root of ionic strength

\section{Discussion}

The sedimentation of HNTs in $\mathrm{NaCl}$ aqueous solution assumes a key role in controlling the colloidal stability with the aim to propose protocols for the separation and purification of nanotubes. Based on literature reports, kaolinite flat particles in $\mathrm{NaCl}$ aqueous solution exhibit a deflocculation up to salt concentration of 0.1-0.15 $\mathrm{M}$ while large aggregate with a edge-toface fabric structure is obtained at large ionic strength due to the screening of the repulsive forces $[3,58]$. Interestingly, despite the coincidence in chemical structure, for HNTs the edgeto-face aggregation cannot be claimed due to the rolling morphological feature of the 
nanotubular clay. On the other hand, at $[\mathrm{NaCl}]>0.01 \mathrm{M}$ the halloysite nanotubes show a clustering effect that follows the reducing of the electrostatic repulsions evidenced by $\zeta$ potential. By comparing the samples from different sources, it comes out the larger $\zeta$-potential variations corresponds to a stronger clustering effect. The increase of $\mathrm{Rh}$ values can be interpreted by considering[59]

$\mathrm{Rh}=\mathrm{L} /\left(2 \sigma-0.19-8.24 / \sigma+12 / \sigma^{2}\right)$

being $L$ the cylinder length and $\sigma=\ln (\mathrm{L} / \mathrm{r})$ with $\mathrm{r}$ the radius of the cylinder. In particular, the variation of Rh by a factor 3 (for HNT_DG) and 4 (for HNT_NZ) going from null ionic strength to the maximum salt concentration (Figure 5) can be obtained by considering a change in the aspect ratio $(\mathrm{L} / \mathrm{r})$ by a factor 0.43 and 0.29 , respectively. In principle, this change reflects a side-by-side arrangement of the nanotubes ( 3 to 4 layers of HNTs in a close compact configuration) as the other possibility related to an end-to-end nanotubes interaction would be entropically unfavorable (ca. 5 HNTs in a row are necessary to justify the observed Rh changes). The differences in settling profiles between the HNT samples from different sources reflects the differences in surface charge and clustering possibilities observed for the two nanotubes. In particular, both clustering and reduced $\zeta$-potential in high salt concentration explain the increase in the settling velocity for HNT_DG with [NaCl]. The HNT_NZ sedimentation velocity is not significantly altered by the ionic strength in agreement with the large $\zeta$-potential that keeps the colloidal stability even in the presence of $\mathrm{NaCl}$. The sedimentation volume fraction is an interesting parameter that is controlled by the repulsive forces exercised between particles in the solvent media [60]. Namely, if the contact distance is approached and the particles remain independent even at the closest packing without sticking together, a significant sedimentation volume is measurable. Due to the significant surface charge the particle-particle repulsive interactions shift to longer range compared to simply hard cylinder systems generating an increasing of the closest average distance between the nanotubes in the sedimentation volume. The significance of the electrostatic forces is clearly 
evidenced by the reduction of the sedimentation volume fraction (Figure 7) generated by the $\mathrm{NaCl}$ addition due to its screening effect. Based on the DLVO theory, the contraction of the ionic double layer width surrounding the nanoparticles is expected to change by 2 order of magnitude if the ionic strength changes from $10^{-4}$ to $1 \mathrm{M}$ [61]. Moreover, high $\mathrm{NaCl}$ concentration enhances the nanotubes clustering and therefore a decrease of the sedimentation volume is expected. By assuming a simple geometrical model and hard cylinder interactions, one can estimate the sedimentation volume fraction (Xs) as [13]

$$
\mathrm{Xs}=\phi_{\mathrm{t}}(\mathrm{L} / \mathrm{r})^{2} / \pi
$$

being $\phi_{t}$ the stoichiometric concentration of nanotubes in volume fraction while $(\mathrm{L} / \mathrm{r})$ is the aspect ratio of the nanotube. This simple approach predicts the differences between the two HNTs samples. Namely, HNT_NZ possesses the largest sedimentation volume ratio in agreement with the larger aspect ratio compared to HNT_DG. At large NaCl concentration the differences in sedimentation volume fraction are cancelled out due to clustering effect.

\section{Conclusions}

We examined the colloidal stability of halloysite nanotubes in water with a special focus on the ionic strength effect. Two samples of natural halloysite nanotubes have been considered from different deposit to which correspond different morphological features. Translational diffusion behavior and $\zeta$-potential evidenced the effect of $\mathrm{NaCl}$ addition to dilute HNTs dispersions. Although qualitatively both nanoclays respond in a similar manner, there are key differences concerning these nanomaterials based on different aspect ratio and effective charge that can influence their colloidal stability. The sedimentation behavior was investigated by time-lapsed technique and a proper image analysis by using a homemade software for the purpose. 
Settling velocity and sedimentation volume are highly dependent on the HNTs sample as well as their dependence on the $\mathrm{NaCl}$ concentration. These aspects can be taken into consideration for designing a proper separation protocol to be used for the separation, purification and standardization of HNTs samples for specific industrial applications.

Acknowledgments. The work was financially supported by Progetto di ricerca e sviluppo "AGM for CuHe" (ARS01_00697) and University of Palermo FFR project MODIFy. 


\section{References}

[1] C. Aguzzi, P. Cerezo, C. Viseras, C. Caramella, Use of clays as drug delivery systems: Possibilities and limitations, Applied Clay Science. 36 (2007) 22-36. doi:10.1016/j.clay.2006.06.015.

[2] E. Ruiz-Hitzky, P. Aranda, M. Darder, G. Rytwo, Hybrid materials based on clays for environmental and biomedical applications, J. Mater. Chem. 20 (2010) 9306-9321.

[3] J. Cao, X. Kang, B. Bate, Microscopic and physicochemical studies of polymer-modified kaolinite suspensions, Colloids and Surfaces A: Physicochemical and Engineering Aspects. 554 (2018) 16-26. doi:10.1016/j.colsurfa.2018.06.019.

[4] E. Paineau, M.S. Amara, G. Monet, V. Peyre, S. Rouzière, P. Launois, Effect of Ionic Strength on the Bundling of Metal Oxide Imogolite Nanotubes, J. Phys. Chem. C. 121 (2017) 21740-21749. doi:10.1021/acs.jpcc.7b07391.

[5] G. Zhuang, J. Gao, H. Chen, Z. Zhang, A new one-step method for physical purification and organic modification of sepiolite, Applied Clay Science. 153 (2018) 1-8. doi:10.1016/j.clay.2017.11.045.

[6] T. Nasim, A. Pal, A. Bandyopadhyay, Flocculation of aqueous kaolin suspension using a biodegradable flocculant system of poly (vinyl alcohol)-Acacia nilotica gum blends, Applied Clay Science. 152 (2018) 83-92. doi:10.1016/j.clay.2017.10.035.

[7] K.C. Etika, L. Liu, M.A. Cox, J.C. Grunlan, Clay-mediated carbon nanotube dispersion in poly(NIsopropylacrylamide), Colloids and Surfaces A: Physicochemical and Engineering Aspects. 489 (2016) 1926. doi:10.1016/j.colsurfa.2015.09.024.

[8] G. Landrou, C. Brumaud, G. Habert, Influence of magnesium on deflocculated kaolinite suspension: Mechanism and kinetic control, Colloids and Surfaces A: Physicochemical and Engineering Aspects. 544 (2018) 196-204. doi:10.1016/j.colsurfa.2017.12.040.

[9] M. Manilo, K. Bohacs, N. Lebovka, S. Barany, Impact of surfactant and clay platelets on electrokinetic potential and size distribution in carbon nanotubes aqueous suspensions, Colloids and Surfaces A: Physicochemical and Engineering Aspects. 544 (2018) 205-212. doi:10.1016/j.colsurfa.2018.02.030.

[10] M.-Y. Wu, Y. Adachi, Effects of electrolyte concentration and $\mathrm{pH}$ on the sedimentation rate of coagulated suspension of sodium montmorillonite, Colloids and Surfaces A: Physicochemical and Engineering Aspects. 506 (2016) 686-693. doi:10.1016/j.colsurfa.2016.07.027.

[11] W. Zhan, H. Yi, S. Song, Y. Zhao, F. Rao, Hydrophobic agglomeration behaviors of clay minerals as affected by siloxane structure, Colloids and Surfaces A: Physicochemical and Engineering Aspects. 568 (2019) 36-42. doi:10.1016/j.colsurfa.2019.01.061.

[12] E. Joussein, S. Petit, G.J. Churchman, B. Theng, D. Righi, B. Delvaux, Halloysite clay minerals - a review, Clay Minerals. 40 (2005) 383-426.

[13] G. Lazzara, G. Cavallaro, A. Panchal, R. Fakhrullin, A. Stavitskaya, V. Vinokurov, Y. Lvov, An assembly of organic-inorganic composites using halloysite clay nanotubes, Current Opinion in Colloid \& Interface Science. 35 (2018) 42-50. doi:10.1016/j.cocis.2018.01.002.

[14] Y. Lvov, W. Wang, L. Zhang, R. Fakhrullin, Halloysite Clay Nanotubes for Loading and Sustained Release of Functional Compounds, Advanced Materials. 28 (2016) 1227-1250. doi:10.1002/adma.201502341.

[15] L. Fu, H. Yang, A. Tang, Y. Hu, Engineering a tubular mesoporous silica nanocontainer with wellpreserved clay shell from natural halloysite, Nano Research. 10 (2017) 2782-2799. doi:10.1007/s12274017-1482-x.

[16] H. Sabahi, M. Khorami, A.H. Rezayan, Y. Jafari, M.H. Karami, Surface functionalization of halloysite nanotubes via curcumin inclusion, Colloids and Surfaces A: Physicochemical and Engineering Aspects. 538 (2018) 834-840. doi:10.1016/j.colsurfa.2017.11.038.

[17] P. Pasbakhsh, G.J. Churchman, J.L. Keeling, Characterisation of properties of various halloysites relevant to their use as nanotubes and microfibre fillers, Appl. Clay Sci. 74 (2013) 47-57. doi:10.1016/j.clay.2012.06.014.

[18] G. Gorrasi, Dispersion of halloysite loaded with natural antimicrobials into pectins: Characterization and controlled release analysis, Carbohydrate Polymers. 127 (2015) 47-53. doi:10.1016/j.carbpol.2015.03.050.

[19] G. Gorrasi, R. Pantani, M. Murariu, P. Dubois, PLA/Halloysite Nanocomposite Films: Water Vapor Barrier Properties and Specific Key Characteristics, Macromolecular Materials and Engineering. 299 (2014) 104115. doi:10.1002/mame.201200424.

[20] M. Du, B. Guo, D. Jia, Newly emerging applications of halloysite nanotubes: a review, Polym. Int. 59 (2010) 574-582.

[21] V. Bertolino, G. Cavallaro, G. Lazzara, S. Milioto, F. Parisi, Halloysite nanotubes sandwiched between chitosan layers: novel bionanocomposites with multilayer structures, New J. Chem. 42 (2018) 8384-8390. doi:10.1039/C8NJ01161C. 
[22] A. Filippov, D. Afonin, N. Kononenko, Y. Lvov, V. Vinokurov, New approach to characterization of hybrid nanocomposites, Colloids and Surfaces A: Physicochemical and Engineering Aspects. 521 (2017) 251-259. doi:10.1016/j.colsurfa.2016.08.079.

[23] E. Abdullayev, Y. Lvov, Clay nanotubes for corrosion inhibitor encapsulation: release control with end stoppers, J. Mater. Chem. 20 (2010) 6681-6687.

[24] A. Joshi, E. Abdullayev, A. Vasiliev, O. Volkova, Y. Lvov, Interfacial Modification of Clay Nanotubes for the Sustained Release of Corrosion Inhibitors, Langmuir. 29 (2013) 7439-7448. doi:10.1021/la3044973.

[25] X. Xing, J. Wang, W. Hu, Q. Li, J. Yuan, Synthesis and inhibition behavior of acid stimuli-responsive CaNa2MoO4-HNTs nanocomposite, Colloids and Surfaces A: Physicochemical and Engineering Aspects. 553 (2018) 305-311. doi:10.1016/j.colsurfa.2018.05.062.

[26] G. Cavallaro, G. Lazzara, L. Lisuzzo, S. Milioto, F. Parisi, Selective adsorption of oppositely charged PNIPAAM on halloysite surfaces: a route to thermo-responsive nanocarriers, Nanotechnology. 29 (2018) 325702.

[27] Y.M. Lvov, M.M. DeVilliers, R.F. Fakhrullin, The application of halloysite tubule nanoclay in drug delivery, Expert Opinion on Drug Delivery. 13 (2016) 977-986. doi:10.1517/17425247.2016.1169271.

[28] M. Massaro, R. Amorati, G. Cavallaro, S. Guernelli, G. Lazzara, S. Milioto, R. Noto, P. Poma, S. Riela, Direct chemical grafted curcumin on halloysite nanotubes as dual-responsive prodrug for pharmacological applications, Colloids and Surfaces B: Biointerfaces. 140 (2016) 505-513. doi:10.1016/j.colsurfb.2016.01.025.

[29] F. Liu, L. Bai, H. Zhang, H. Song, L. Hu, Y. Wu, X. Ba, Smart H2O2-Responsive Drug Delivery System Made by Halloysite Nanotubes and Carbohydrate Polymers, ACS Appl. Mater. Interfaces. 9 (2017) 31626 31633. doi:10.1021/acsami.7b10867.

[30] H. Zhang, C. Cheng, H. Song, L. Bai, Y. Cheng, X. Ba, Y. Wu, A facile one-step grafting of polyphosphonium onto halloysite nanotubes initiated by $\mathrm{Ce}(\mathrm{iv})$, Chem. Commun. (2019). doi:10.1039/C8CC08667B.

[31] E. Rozhina, S. Batasheva, M. Gomzikova, E. Naumenko, R. Fakhrullin, Multicellular spheroids formation: The synergistic effects of halloysite nanoclay and cationic magnetic nanoparticles, Colloids and Surfaces A: Physicochemical and Engineering Aspects. 565 (2019) 16-24. doi:10.1016/j.colsurfa.2018.12.038.

[32] G. Cavallaro, G. Lazzara, S. Milioto, F. Parisi, F. Ruisi, Nanocomposites based on esterified colophony and halloysite clay nanotubes as consolidants for waterlogged archaeological woods, Cellulose. 24 (2017) 3367-3376. doi:10.1007/s10570-017-1369-8.

[33] G. Cavallaro, S. Milioto, F. Parisi, G. Lazzara, Halloysite Nanotubes Loaded with Calcium Hydroxide: Alkaline Fillers for the Deacidification of Waterlogged Archeological Woods, ACS Appl. Mater. Interfaces. 10 (2018) 27355-27364. doi:10.1021/acsami.8b09416.

[34] G. Cavallaro, G. Lazzara, S. Milioto, F. Parisi, Halloysite Nanotubes for Cleaning, Consolidation and Protection, The Chemical Record. 18 (2018) 940-949. doi:10.1002/tcr.201700099.

[35] G. Cavallaro, S. Milioto, L. Nigamatzyanova, F. Akhatova, R.F. Fakhrullin, G. Lazzara, Pickering Emulsion Gels Based on Halloysite Nanotubes and Ionic Biopolymers: Properties and Cleaning Action on Marble Surface, ACS Appl. Nano Mater. (2019). doi:10.1021/acsanm.9b00487.

[36] G. Cavallaro, G. Lazzara, S. Milioto, F. Parisi, V. Sparacino, Thermal and dynamic mechanical properties of beeswax-halloysite nanocomposites for consolidating waterlogged archaeological woods, Polymer Degradation and Stability. 120 (2015) 220-225. doi:10.1016/j.polymdegradstab.2015.07.007.

[37] S. Sadjadi, M.M. Heravi, M. Malmir, Pd@HNTs-CDNS-g-C3N4: A novel heterogeneous catalyst for promoting ligand and copper-free Sonogashira and Heck coupling reactions, benefits from halloysite and cyclodextrin chemistry and g-C3N4 contribution to suppress Pd leaching, Carbohydrate Polymers. 186 (2018) 25-34. doi:10.1016/j.carbpol.2018.01.023.

[38] S. Sadjadi, M. Atai, Ternary hybrid system of halloysite nanotubes, polyacrylamides and cyclodextrin: an efficient support for immobilization of $\mathrm{Pd}$ nanoparticles for catalyzing coupling reaction, Applied Clay Science. 153 (2018) 78-89. doi:10.1016/j.clay.2017.12.013.

[39] A. Glotov, A. Stavitskaya, Y. Chudakov, E. Ivanov, W. Huang, V. Vinokurov, A. Zolotukhina, A. Maximov, E. Karakhanov, Y. Lvov, Mesoporous Metal Catalysts Templated on Clay Nanotubes, Bulletin of the Chemical Society of Japan. 92 (2019) 61-69. doi:10.1246/bcsj.20180207.

[40] V.A. Vinokurov, A.V. Stavitskaya, Y.A. Chudakov, E.V. Ivanov, L.K. Shrestha, K. Ariga, Y.A. Darrat, Y.M. Lvov, Formation of metal clusters in halloysite clay nanotubes, Science and Technology of Advanced Materials. 18 (2017) 147-151. doi:10.1080/14686996.2016.1278352.

[41] Y. Liu, H. Guan, J. Zhang, Y. Zhao, J.-H. Yang, B. Zhang, Polydopamine-coated halloysite nanotubes supported AgPd nanoalloy: An efficient catalyst for hydrolysis of ammonia borane, International Journal of Hydrogen Energy. 43 (2018) 2754-2762. doi:10.1016/j.ijhydene.2017.12.105. 
[42] Q. Wang, Y. Wang, Y. Zhao, B. Zhang, Y. Niu, X. Xiang, R. Chen, Fabricating roughened surfaces on halloysite nanotubes via alkali etching for deposition of high-efficiency Pt nanocatalysts, CrystEngComm. 17 (2015) 3110-3116. doi:10.1039/C5CE00189G.

[43] Y. Liu, J. Zhang, H. Guan, Y. Zhao, J.-H. Yang, B. Zhang, Preparation of bimetallic Cu-Co nanocatalysts on poly (diallyldimethylammonium chloride) functionalized halloysite nanotubes for hydrolytic dehydrogenation of ammonia borane, Applied Surface Science. 427 (2018) 106-113. doi:10.1016/j.apsusc.2017.08.171.

[44] G. Cavallaro, I. Grillo, M. Gradzielski, G. Lazzara, Structure of Hybrid Materials Based on Halloysite Nanotubes Filled with Anionic Surfactants, J. Phys. Chem. C. 120 (2016) 13492-13502. doi:10.1021/acs.jpcc.6b01282.

[45] V. Bertolino, G. Cavallaro, G. Lazzara, S. Milioto, F. Parisi, Biopolymer-Targeted Adsorption onto Halloysite Nanotubes in Aqueous Media, Langmuir. 33 (2017) 3317-3323. doi:10.1021/acs.langmuir.7b00600.

[46] M. Liu, R. He, J. Yang, W. Zhao, C. Zhou, Stripe-like Clay Nanotubes Patterns in Glass Capillary Tubes for Capture of Tumor Cells, ACS Appl. Mater. Interfaces. 8 (2016) 7709-7719. doi:10.1021/acsami.6b01342.

[47] I. McDOWALL, W. VOSE, Sedimentation of Halloysite, Nature. 170 (1952) 368-368. doi:10.1038/170368a0.

[48] Z. Luo, H. Song, X. Feng, M. Run, H. Cui, L. Wu, J. Gao, Z. Wang, Liquid Crystalline Phase Behavior and Sol-Gel Transition in Aqueous Halloysite Nanotube Dispersions, Langmuir. 29 (2013) 12358-12366. doi:10.1021/la402836d.

[49] P.R. Chang, Y. Xie, D. Wu, X. Ma, Amylose wrapped halloysite nanotubes, Carbohydr. Polym. 84 (2011) 1426-1429. doi:doi: 10.1016/j.carbpol.2011.01.038.

[50] G. Cavallaro, G. Lazzara, S. Milioto, F. Parisi, V. Sanzillo, Modified Halloysite Nanotubes: Nanoarchitectures for Enhancing the Capture of Oils from Vapor and Liquid Phases, ACS Appl. Mater. Interfaces. 6 (2014) 606-612. doi:10.1021/am404693r.

[51] L. Lisuzzo, G. Cavallaro, F. Parisi, S. Milioto, G. Lazzara, Colloidal stability of halloysite clay nanotubes, Ceramics International. 45 (2019) 2858-2865. doi:10.1016/j.ceramint.2018.07.289.

[52] N. Kiryati, Y. Eldar, A.M. Bruckstein, A probabilistic Hough transform, Pattern Recognition. 24 (1991) 303-316. doi:10.1016/0031-3203(91)90073-E.

[53] G. Cavallaro, L. Chiappisi, P. Pasbakhsh, M. Gradzielski, G. Lazzara, A structural comparison of halloysite nanotubes of different origin by Small-Angle Neutron Scattering (SANS) and Electric Birefringence, Applied Clay Science. 160 (2018) 71-80. doi:10.1016/j.clay.2017.12.044.

[54] R.C. Gonzalez, R.E. Woods, B.R. Masters, Digital Image Processing, Third Edition, Journal of Biomedical Optics. 14 (2009) 029901. doi:10.1117/1.3115362.

[55] L.A.F. Fernandes, M.M. Oliveira, Real-time line detection through an improved Hough transform voting scheme, Pattern Recognition. 41 (2008) 299-314. doi:10.1016/j.patcog.2007.04.003.

[56] P. Mukhopadhyay, B.B. Chaudhuri, A survey of Hough Transform, Pattern Recognition. 48 (2015) 9931010. doi:10.1016/j.patcog.2014.08.027.

[57] D. Cascio, V. Taormina, G. Raso, An Automatic HEp-2 Specimen Analysis System Based on an Active Contours Model and an SVM Classification, Applied Sciences. 9 (2019) 307. doi:10.3390/app9020307.

[58] A.M. Palomino, J.C. Santamarina, Fabric map for kaolinite: effects of pH and ionic concentration on behavior, Clays and Clay Minerals. 53 (2005) 211-223. doi:10.1346/CCMN.2005.0530302.

[59] K. Matsuoka, A. Yonekawa, M. Ishii, C. Honda, K. Endo, Y. Moroi, Y. Abe, T. Tamura, Micellar size, shape and counterion binding of N-(1,1-Dihydroperfluoroalkyl)-N,N,N-trimethylammonium chloride in aqueous solutions, Colloid and Polymer Science. 285 (2006) 323-330. doi:10.1007/s00396-006-1574-8.

[60] H. Freundlich, A.D. Jones, Sedimentation Volume, Dilatancy, Thixotropic and Plastic Properties of Concentrated Suspensions., J. Phys. Chem. 40 (1935) 1217-1236. doi:10.1021/j150378a012.

[61] B.V. Derjaguin, L.D. Landau, Theory of the Stability of Strongly Charged Lyophobic Sols and of the Adhesion of Strongly Charged Particles in Solutions of Electrolytes., Acta Physicochim. 14 (1941) 733762. 